\title{
Editorial
}

\section{Towards a More Complete Standardization of Mite Allergen Extracts}

\author{
Enrique Fernández-Caldas
}

Inmunotek S.L., Madrid, Spain

Dermatophagoides pteronyssinus and D. farinae are among of the most important sources of clinically relevant allergens worldwide. Mites are cultured in the laboratory and the material harvested from large scale cultures is used to prepare mite extracts for diagnosis, immunotherapy of mite-allergic individuals and allergy and immunology research. Kilogram quantities of mite cultures are harvested yearly and millions of individuals are diagnosed with allergen extracts and treated with mite vaccines.

Although there are some general recommendations on the subject, mite cultures may be used as whole cultures, thus containing more fecal material, or sieved, containing more purified mites. Both raw materials are used. Currently, there is a clear trend towards using more purified cultures to avoid the presence of food medium. The use of a well-defined nonallergic medium is also warranted. $D$. pteronyssinus and $D$. farinae have a global distribution. The allergenicity of other species, such as Blomia tropicalis, Lepidoglyphus destructor and Tyrophagus putrescenti$a e$, has also been demonstrated. Extracts of these species are also commercially available in many countries.

Although the allergenicity of house dust was known for many years, only in the mid-1960s did it become clear that house dust mites were the main source of house dust mite allergens. Although mites were first grown on human skin scales collected from barber shops and on yeast [1], other food media used included fish food flakes, dried Daphnia, dog food, rodent chow, several cereal preparations, and even mold cultures [2]. Due to their position in the trophic chain, mites mainly feed on proteins found in house and mattress dust. A supplement of yeast is also important to complement the intake of micronutrients. The ingestion of a high-protein diet is a common determinant and is needed for their proliferation.

Several food media are currently being used to grow mites. There are general recommendations to avoid the use of human-, and other animal-, derived proteins. Food media currently used in Europe and the USA include autoclaved pork liver powder and yeast, brine shrimp eggs and yeast and wheat germ. Other companies produce mites grown on wheat germ supplemented with amino acids (resembling the composition of human stratum corneum) and baker's yeast [3].

For the preparation of allergen extracts, the United States Food and Drug Administration (FDA) only allows the use of mite cultures containing more than, or at least, 99\% pure mites (see FDA website). In Europe, there are also general recommendations by the European Medicines Agency [4], which state: 'The cultivation method

\section{KARGER}

Fax +4161306 1234

E-Mail karger@karger.ch

www.karger.com
(C) 2012 S. Karger AG, Basel

$1018-2438 / 13 / 1601-0001 \$ 38.00 / 0$

Accessible online at:

www.karger.com/iaa
Correspondence to: Dr. Enrique Fernández-Caldas

Inmunotek S.L.

Avenida Somosierra 22, N14A

ES-28700 San Sebastián de los Reyes, Madrid (Spain)

Tel. +34 685974 517, E-Mail efcaldas@inmunotek.com 
and the composition of the cultivation medium as well as the media components should be described. Details on the composition of the cultivation medium and the media components should be submitted. Synthetic and consequently free of animal-derived material and allergen-free media should be preferably used. The conditions of culture and the time of harvest should be described and the corresponding key parameters defined. It should be indicated which part of the culture is used for further processing, e.g. mites, mite feces only or the whole mite culture or mixes thereof.' This sentence implies that consecutive batches of the mite source materials should be similar in composition to assure the consistency and allergen composition of the produced vaccines.

The study by Avula-Poola et al. [5] demonstrates that $D$. farinae and D. pteronyssinus cultures grow at different rates. They also demonstrate that rates of accumulation and the amounts of group 1 and group 2 allergens and the levels of endotoxin in mite cultures, and in the extracts prepared from them, are dramatically influenced by the foods on which the mites were grown. The differences in the population growth and allergen accumulation dynamics between $D$. farinae and $D$. pteronyssinus grown on the two different diets were not unexpected since ecological and laboratory studies have clearly shown that the two species have different biological requirements. Laboratory studies at comparable relative humidity and temperature conditions show differences in feeding, fecundity, reproduction, population growth rates and survival between the two species. Similar results have also been observed in longitudinal studies [6]. The two diets used in this study (diet A: 9:1 mixture of dried whole egg and baker's yeast and diet B: 9:1 mixture of laboratory rodent chow and baker's yeast) further illustrate the biological differences between the two species and how growth media influence the efficiency of producing mites and the allergen content of culture material. Thus, diet should be an important consideration when culturing mites and comparing allergen data of mite extracts from different sources and in standardizing and characterizing mite extracts [7].

This new information is relevant for the preparation of mite extracts. It may open the door for a more strict standardization procedure of the raw materials used in the preparation of allergen extracts and suggests that a more precise approach is possible. However, important issues still remain unanswered such as the following: are mite cultures which contain larger amounts of spent medium (more fecal material) more clinically effective to diagnose, or treat, mite-allergic individuals? Is there a preferred Der $\mathrm{p}$ 1/Der $\mathrm{p} 2$ ratio? What is the clinical contri- bution of other mite allergens to the diagnosis and treatment of mite allergies?

Allergen standardization provides the basis for better and more reproducible allergen vaccines. In recent years, great efforts have been made to offer better standardized extracts. The selection of raw materials remains a key issue for mite allergen extracts. Currently, mites are being commercially grown on a variety of food substrates, and the purity of the mite bodies in the total extract varies among the different sources and different countries. It could be argued that considering that patients inhale mite feces, and supposedly not mite bodies, mite allergen extracts containing just mite feces would be more appropriate to diagnose and treat. However, little is known about the clinical impact of using whole mite cultures, purified feces, or just pure mite bodies. While in the USA, the use of pure mite bodies is mandatory, to minimize food medium contamination, in Europe, both types of mite raw materials are being offered and used.

Several publications have demonstrated that mite allergen extracts for diagnosis and treatment produced in different countries may vary in their allergen concentration and composition [8]. However, fewer studies have demonstrated that different mite extracts, produced from different raw materials, may induce different immunological responses in vivo and in vitro [9]. In this study, 3 house dust mite extracts, prepared from pure mite bodies, were analyzed for endotoxin levels, protease and chitinase activities and effects on transepithelial resistance, junctional proteins and proinflammatory cytokine release. The different extracts varied extensively in their biochemical properties and induced divergent responses in vitro and in vivo. No information was provided on the diet on which these mites were grown. This study also stresses the importance to properly standardize mite allergen extracts for immunological studies, since extracts from the same species may produce divergent results.

In summary, the article by Avula-Poola et al. [5] clearly shows important differences when mites are grown on dissimilar media. It also shows that differences in endotoxin and major allergen content are also a consequence of differences in the diet. Mite allergen extracts are a complex mixture of proteins with several biological activities. More studies should focus on the influence of the food medium on allergen production rates, enzymatic activity and total allergenicity. The role of the diet on the expression of allergens should be further investigated to standardize the production process of mite source materials for more representative and clinically effective mite vaccines and allergen extracts. 


\section{References}

-1 Spieksma FHM, Spieksma-Boezeman MIM: The mite fauna of house dust with particular reference to the house-dust mite D. pteronyssinus (Trt.) (Psoroptidae: Sarcoptiformes). Acarologia 1967;9:226-241.

2 Colloff MJ: Dust Mites. Collingwood, CSIRO Publishing, 2009, pp 268-271.

$\checkmark 3$ Batard T, Hrabina A, Bi XZ, Chabre H, Lemoine P, Couret M-N, et al: Production and proteomic characterization of pharmaceutical-grade Dermatophagoides pteronyssinus and Dermatophagoides farinae extracts for allergy vaccines. Int Arch Allergy Immunol 2006;140:295-305.

4 Guideline on allergen products: production and quality issues. Committee for Medicinal Products for Human Use (CHMP). European Medicines Agency Evaluation of Medicines for Human Use. London, 20 November 2008, EMEA/CHMP/BWP/304831/2007.
Avula-Poola S, Morgan MS, Arlian LG: Diet influences growth rates and allergen and endotoxin contents of cultured Dermatophagoides farinae and Dermatophagoides pteronyssinus house dust mites. Int Arch Allergy Immunol 2012;159:226-234.

6 Fernández-Caldas E, Andrade J, Trudeau WL, Souza Lima E, Souza Lima I, Lockey RF: Serial determinations of Der $\mathrm{p} 1$ and Der $\mathrm{f} 1$ show predominance of one Dermatophagoides species. J Investig Allergol Clin Immunol 1998;8:27-29.
Casset A, Mari A, Purohit A, Resch Y, Weghofer M, Ferrara R, Thomas WR, Alessandri C, Chen K-W, de Blay F, Valenta R, Vrtala S: Varying allergen composition and content affects the in vivo allergenic activity of commercial Dermatophagoides pteronyssinus extracts. Int Arch Allergy Immunol 2012;159: 253-262.

8 Burazer L, Milovanovic K, Milovanovic M, Vuckovic O, Velickovic TC, GavrovicJankulovic M: Impact of Dermatophagoides pteronyssinus mite body raw material on house dust mite allergy diagnosis in a Serbian population. Med Vet Entomol 2011;25: 77-83.

$\checkmark 9$ Post S, Nawijn MC, Hackett TL, Baranowska M, Gras R, van Oosterhout AJ, Heijink IH: The composition of house dust mite is critical for mucosal barrier dysfunction and allergic sensitisation. Thorax 2012;67:488495 